\title{
Ableitung der absoluten Konfiguration von Alkaloiden der Aspidospermingruppe durch optischen Vergleich mit Alkaloiden der Strychningruppe
}

In einer kürzlich veröffentlichten Mitteilung 1 wurde durch chemische Korrelation mit den in ihrer absoluten Konfiguration bekannten stark linksdrehenden Alkaloiden 19,20-Dihydroakuammicin (I) und Tubifolin (II) gezeigt, dass den stark rechtsdrehenden Basen Tubotaiwin (=19, 20-Dihydrocondylocarpin) (III) und Condyfolin (IV) die nachstehende absolute Konfiguration zukommt.

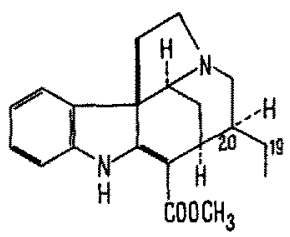

19,20-Dihydroakuammicin (I) $[\mathrm{M}]_{\mathrm{D}}^{\mathrm{CH}_{\mathrm{a}} \mathrm{OH}}-2065^{\circ}$

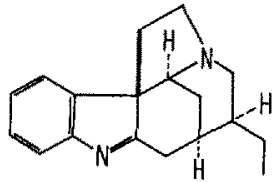

Tubifolin (II); $[\mathrm{M}]_{\mathrm{D}}^{\mathrm{C}_{2} \mathrm{H}_{4} \mathrm{OH}}-831^{\circ}$, in $\mathrm{CHCl}_{\mathrm{g}}-911^{\circ}$, in Essigester $-962^{\circ}$

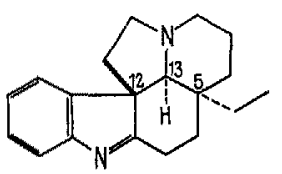

(-)-1,2-Dehydroaspidospermidin $(V):[M]_{\mathrm{D}}^{\mathrm{C}_{2} \mathrm{H}_{5} \mathrm{OH}}-681^{\circ}$

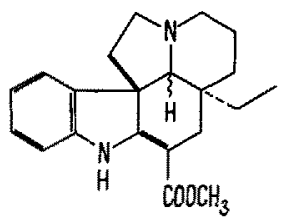

(-)-Vincadifformin (VIII) $[\mathrm{M}]_{\mathrm{D}}^{\mathrm{C}_{2} \mathrm{H}_{5} \mathrm{OH}}-1821^{\circ}$

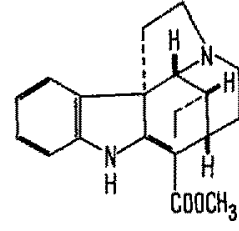

Tubotaiwin (III) $[\mathrm{M}]_{\mathrm{D}}^{\mathrm{CHCl}}+1936^{\circ}$

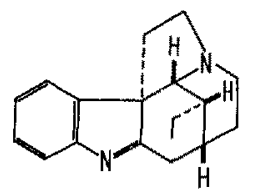

Condyfolin (IV)

$[M]_{D}^{\text {Essigester }}+927^{\circ}$

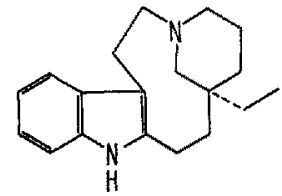

$(+)$-Quebrachamin (VI)

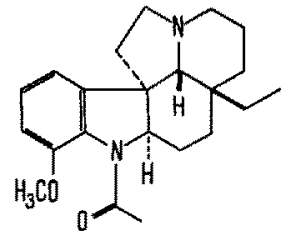

(-)-Aspidaspermin (VII)
Strukturell und damit in ihrem molekularen Bau sehr nahe verwandt mit Tubifolin und Condyfolin sind das stark rechts- bzw, linksdrehende $(+)^{2_{-}}$und $(-)^{3}-1,2$ Dehydroaspidospermidin (V) ${ }^{4}$.

(-)-V zeigt eine ähnliche Molekularrotation wie Tubifolin und der (bisher in der Natur noch nicht angetroffene) Antipode von Condyfolin. Diese drei Stoffe besitzen dasselbe chromophore Zentrum. Wo dieses Zentrum auch immer ist, es lassen sich daran räumliche Oktanden anlegen, die in allen drei Verbindungen die gleichen zum Zentrum nächstgelegenen Substituenten in den gleichen Oktanden enthalten. Dem (-)-1,2-Dehydroaspidospermidin lässt sich daher die absolute Konfiguration V (dem rechtsdrehenden die spiegelbildliche) zuschreiben.

Die Bildung von $(+)$ Quebrachamin ${ }^{2,3}$ aus $(-)-V$ zeigt, dass dem ersteren die absolute Stereochemie VI zukommt. (-)-Aspidospermin besitzt am C-5 die gleiche absolute Konfiguration wie (-)-Quebrachamin ${ }^{6}$, so dass thm die absolute Konfiguration VII zugeschrieben werden kann.

(-)-Vincadifformin ${ }^{3}\left([M]_{D}=-1821^{\circ}\right)$ geht durch $\mathrm{De}$ carbomethoxylierung in $(-)-V$ uber und besitzt daher die absolute Konfiguration VIII ${ }^{6}$.

Zur Zeit wird versucht, durch RD-Messungen unter Heranziehung weiterer Relais-Substanzen den vorgeschlagenen konfigurativen Zusammenhang zwischen Strychnin- und Aspidosperminalkaloiden zu erhärten ${ }^{7}$.

Summary. The absolute configuration of certain alkaloids of the Aspidospermin group is suggested.

D. SCHUMANN,

B. W. BYcrofr and H. SCHMID

Organish-Chemisches Institut der Universität, Zürich (Schveiz), 21. Dezember 1963.

t D. Schumann und H. Schmid, Helv, chim. Acta 46, 1996 (1963).

2 G. F. Smith und M. A. Wahid, J. chem. Soc. 1963, 4002. Das (t)1, 2-Dehydroaspidospermidin zeigt $[\alpha]_{D}^{\mathrm{C}_{8} \mathrm{H}_{8} \mathrm{OH}}=+243^{\circ}$.

3 M. Plat, J. Le Men, M.-M. Janot, J. M. Wilson, H. Budzikiewicz, L. J. Durham, Y. Nakagawa und C. Djerassi, Tetrahedron Letters No. 7,271 (1962). (-)-1,2-Dehydroaspidospermidin zeigt $[\alpha]_{D}^{\mathrm{C}_{2} \mathrm{H}_{8} \mathrm{OH}}=-225^{\circ}$.

4 Für die rclative, der stabilsten Anordnung entsprechende Kon figuration dieses Alkaloids existieren gute Argumente: ${ }^{2}$, sowie G. Stork und J. E. Dolfini, J. Amer. chem. Soc. 85, 2872 (I963) (liussnote 9).

5 K. Bremann und G. Sptteller, J. Amer, chem. Soc. 84, 4578 (1962).

- Die französischen und amerikanischen Autoren haben auf Grund von NMR-Argumenten für das Alkaloid das gespannte System mit cis-Stellung des $\mathrm{H}$-Atoms am $\mathrm{C}-13$ und der Athylenbrücke am $\mathrm{C}-12$ vorgeschlagen, was die Molekulgestalt gegenüber einer trans-Anordnung deutlich verändert; vgl. hierzu?

7 Dem Schweizerischen Nationalfonds danken wir für Unterstützung.

\section{5-Azacytidine, a New, Highly Effective Cancerostatic}

In the course of studies on the azapyrimidines as potential inhibitors of nucleic acid biosynthesis, a method for the synthesis of nucleosides derived from 5-azacytosine has recently been developed in the laboratories".

Biological testing of these compounds has revealed that the cytidine analogue, 5-azacytidine (5-AzCR, m.p. 230 ), has significant antimetabolic and cancerostatic effects.
The substance, in very small concentrations, inhibits the growth of some bacteria. Thus the growth of $E$. coli $\mathrm{B}$ in a synthetic medium is inhibited to the extent of $50 \%$ at concentrations of $0.25 \mu \mathrm{g} \mathrm{5-AzCR}$ per $\mathrm{ml}$, in strong contrast to a weak bacteriostatic effect $(50 \%$ growth inhibition at $520 \mu \mathrm{g}$ per $\mathrm{ml}$ ) shown by the 6 -azacytidine which also

1 F. Sorm and A. Pískata, Coll. Czech. chem. Commun., in press. Czechoslovak patent application No. PV 5916-63 and PV 7093-63. 